geget (2019) Volume 14 - Issue1

Original Article

\title{
Rate and Type of Infections in Children with Nephrotic syndrome
}

\author{
Doaa Mohammed Youssef, Ali Mohammed Abo Zeid, Mona Shaaban Ali Selim, \\ Moataz Mohamed Sallam \\ Departments of Pediatrics, Faculty of Medicine, Zagazig University
}

\begin{abstract}
Introduction

Changes in immunity are complex in idiopathic nephrotic syndrome (NS), affecting the cellular and the humoral balance of specific immunity which may impair the response to infection, so at least half of relapses triggered by infection. We aimed to describe the rate and type of infection in idiopathic nephrotic syndrome cases admitted with activity to our Nephrology unit
\end{abstract}

\section{Patients and methods}

This is a cross-sectional, case control study; 90 NS cases were admitted with activity, they were subdivided in to two groups according to presence or absence of infection; group I $60 \mathrm{NS}$ cases with infection and group II $30 \mathrm{NS}$ cases without infection. We evaluated every subject for the activity of disease, type of infections, the occurrence of complications and fate of therapy and routine laboratory tests were done.

\section{Result}

The most common type of infection was gastroenteritis (GE) (25\%) of infected cases, 2nd most common was Urinary tract infection (UTI) (21.7\%), While Pneumonia representatives (15\%) of infection. There was no significant pathogen in $(61.66 \%)$ of all infected cases, the most common pathogen is E.coli (25\%), followed by Shigella $(6.66 \%)$, pneumococci $(3.33 \%)$, Klebsiella (1.66\%) and Proteus (1.66\%). The most often used antibiotic before culture is (Ampicillin, sulbactam followed by cefotaxime) and after culture (Cefepime, Meronem, ceftazidime and Vancomycin).

\section{Conclusion}

We concluded that $66.6 \%$ of admitted children with relapsed NS were due to infection, the most common type of infection is GE, followed by UTI, Viral infection represents were more common than bacterial ones, the most common isolated bacteria after culture were E.coli, Shigell and pneumococci.

\section{Keywords}

Common, infections, childhood, nephrotic

\section{Correspondence}

Doaa Mohammed Youssef

Faculty of Medicine, Zagazig University,

Email: Dody5176@yahoo.com

ORCID No: 0000-0001-9948-5690

Postal code: 11258

\author{
geget : The Journal of the Egyptian Society of Pediatric Nephrology and Transplantation (ESPNT) \\ geget https://geget.journals.ekb.eg/ \\ Published by ESPNT http://espnt.net/ \\ Cohosted by Egyptian Knowledge Bank https://www.ekb.eg
}




\section{Introduction}

Nephrotic syndrome is a disease affects the kidney exclusively and is marked typically by the effacement of podocyte foot processes without glomerular deposit or inflammatory lesion [1]. Children with NS acquire many acute complications some of which may be serious and fatal; as infections, venous thromboembolism (VTE), and AKI, when clinical reflections of infection and VTE on nephrotic children are clear, the epidemiology and fate of AKI remain vague [2].

Without treatment, nephrotic child is more prone to mortality, mostly due to bacterial infection. Before era of corticosteroids and antibiotics, $40 \%$ of children died, with $50 \%$ of these losses due to infection [3]. Recently it is proved that at least $50 \%$ of activities in pediatric onset NS are stimulated by a viral upper respiratory tract infection; this may be due to non-specific host response to infection more than to virus itself or their antibody response.

Thus, other infections such as urinary tract infection (UTI), diarrhea, peritonitis and skin infections have also been implicated. Many researches have proved the role of UTI as an important trigger of relapse, and the cause of steroid resistance. Thus; many studies have found specific initiators of activates, targeted interventions have been shown to modify disease course [4]. Children with nephrotic syndrome who respond to therapy have an $80-$ $90 \%$ possibility of having one or more activates. $50 \%$ of those that have activity are infrequent relapses and can be managed with short courses of prednisone [3]. Our aim was to describe the accurate rate of infection and specific type of treatment in nephrotic syndrome cases admitted with activity to nephrology unit.

\section{Patients and methods}

This is a cross-sectional, case control study performed among admitted nephrotic syndrome cases with activity to inpatient pediatric nephrology unit, Zagazig University Hospitals. 90 subjects participated in this study; these were selected from the date of starting the study in July 2016 up till fulfilling the sample size in October 2016, they were divided into two group I 60 admitted with infection and group II 30 nephrotic syndrome admitted without infection.

This study was approved from the Ethical Committee in Faculty of Medicine, Zagazig University. Written informed consent was taken from the study subjects (infected group+ non infected group and their parents). We concluded Admitted nephrotic cases with activity or relapse, Age ranged from birth to 18years, both sexes were included and all social and educational classes.

We excluded; admitted cases with no activity, activity without admission and those who refused participation. Participants were subjected to a questionnaire which specially designed to collect; Socio-demographic data including age, sex, Clinical data including: age of onset of nephrotic syndrome, duration of illness ,number of relapses, response to steroid, types and duration of any medication including specific treatment as regarding antibiotic and anti-viral treatment, supportive treatment. All cases with nephrotic syndrome were evaluated for the activity of disease, type of infections, occurrence of complications and fate of therapy. Laboratory tests were done including) CBC, serum creatinine, BUN, Total serum protein, serum albumin and CRP).

Statistical analysis: Data were then imported into Statistical Package for the Social Sciences (SPSS version 20.0) (Statistical Package for the Social Sciences) software for analysis.

\section{Results}

90 child with nephrotic syndrome were admitted with relapse in our nephrology unit during the period of our study; they were group A 60 cases (44 males, 16 females) admitted with infection their mean age $6.66 \pm 4.6$ years old, and group B 30 (21 males, 9 females) without infection with mean age $5.15 \pm 3$ years old. The most common type of infection was gastroenteritis (GE) (25\%) of infected cases and; [ it was caused by E.coli (33\%), viral infection $(40 \%)$, Shigella $(20 \%)$, and no pathogen in $(6.7 \%)$ of gastroenteritis cases], 2nd most common Then Urinary tract infection (UTI) (21.7\%); [it was caused by E.coli $(64.2 \%)$, viral $(23.1 \%)$, no pathogen in $(15.4 \%)$, proteus $(7.7 \%)$ and klebsiella in $(7.7 \%)]$, While Pneumonia represented $(15 \%)$ of infection; [ it was caused by viral infection (55.6\%), no pathogen in $(33.3 \%)$ and pneumococci in (11.1\%)], Upper respiratory tract infection (URTI) also represented (15\%) of cases, no pathogen in $(55.6 \%)$, viral infection in $(44.6 \%)$.

We found also Peritonitis represented $(1.7 \%)$ of infection it was caused by viral infection. Other cases were admitted with more than one site of infection as shown in table (1). Table 1 also showed that there was no significant pathogen in $(61.66 \%)$, the most common pathogen was E.coli $(25 \%)$, followed by Shigella $(6.66 \%)$, pneumococci $(3.33 \%)$, klebsiella $(1.66 \%) \&$ proteus $(1.66 \%)$ of all infected cases We found leukocytosis in (28.3\%), Neutropenia in $(26.7 \%)$ \& Lymphocytosis in $(21.7 \%)$ of infected cases combined lymphocytosis, neutropenia and negative CRP results in $(21.7 \%)$ of cases. 
Table 1: Types of infection and culture results in infected group.

\begin{tabular}{|c|c|c|c|c|c|c|c|c|c|}
\hline \multirow{3}{*}{ Type of infection } & \multirow{3}{*}{ No } & \multirow{3}{*}{$\%$} & \multirow{3}{*}{$\begin{array}{l}\text { Sample of } \\
\text { culture }\end{array}$} & \multicolumn{6}{|c|}{ Result of culture } \\
\hline & & & & \multicolumn{2}{|c|}{-ve $\quad N=13$} & \multicolumn{2}{|l|}{ +ve $\quad N=21$} & \multicolumn{2}{|c|}{ Viral $\quad \mathbf{N}=\mathbf{2 6}$} \\
\hline & & & & No & $\%$ & No & $\%$ & No & $\%$ \\
\hline \multirow{2}{*}{ GE } & \multirow{2}{*}{15} & \multirow{2}{*}{25} & \multirow{2}{*}{ Stools } & \multirow{2}{*}{1} & \multirow{2}{*}{6.7} & E.coli (5) & 33.3 & \multirow{2}{*}{6} & \multirow{2}{*}{40} \\
\hline & & & & & & Shigella (3) & 20 & & \\
\hline \multirow{3}{*}{ UTI } & \multirow{3}{*}{13} & \multirow{3}{*}{21.7} & \multirow{3}{*}{ Urine } & \multirow{3}{*}{2} & \multirow{3}{*}{15.4} & E.coli (6) & 46.2 & \multirow{3}{*}{3} & \multirow{3}{*}{23.1} \\
\hline & & & & & & Proteus (1) & 7.7 & & \\
\hline & & & & & & Klebsiella (1) & 7.7 & & \\
\hline Pneumonia & 9 & 15 & Sputum & 3 & 33.3 & Pneumococci (1) & 11.1 & 5 & 55.6 \\
\hline URTI & 9 & 15 & Sputum & 5 & 55.6 & - & 0.0 & 4 & 44.4 \\
\hline Peritonitis & 1 & 1.7 & Bl. culture & - & 0.0 & - & 0.0 & 1 & 100 \\
\hline GE +UTI & 3 & 5 & Urine & - & 0.0 & E.coli (2) & 66.7 & 1 & 33.3 \\
\hline Pneumonia + UTI & 2 & 3.3 & Urine & 2 & 100 & - & 0.0 & - & 0.0 \\
\hline $\begin{array}{l}\text { Pneumonia+ } \\
\text { Peritonitis }\end{array}$ & 2 & 3.3 & Bl. culture & - & 0.0 & - & 0.0 & 2 & 100 \\
\hline GE + URTI & 1 & 1.7 & Stools & - & 0.0 & - & 0.0 & 1 & 100 \\
\hline GE + Pneumonia & 2 & 3.3 & Stools & - & 0.0 & Shigella (1) & 50 & 1 & 50 \\
\hline GE + Frunculosis & 1 & 1.7 & Stools & - & 0.0 & - & 0.0 & 1 & 100 \\
\hline GE + Peritonitis & 2 & 3.3 & Stools & - & 0.0 & E.coli (1) & 50 & 1 & 50 \\
\hline
\end{tabular}

GE gastroenteritis, UTI Urinary tract infection, URTI Upper respiratory tract infection.

We reported that the most frequently used antibiotic before culture is Ampicillin, sulbactam followed by cefotaxime and after culture Cefepime, Meronem, ceftazidime and Vancomycin are the most frequently used table (2), while we used antiviral therapy in 13 cases $21.7 \%$ of infected cases. all cases received cyclosporine were presented with active disease, While not all cases received azathioprine presented with active disease; that means that cyclosporine precipitate infection table (3). We found that $(91.7 \%)$ of infected group had fair out come and $(8.3 \%)$ of cases had unfavorable outcome as following: (5\%) of cases show Activity leading to renal failure, $(1.7 \%)$ of cases died from pneumonia and $(1.7 \%)$ of cases show thrombosis. 
Table 2: antibiotic therapy used to treat infection.

\begin{tabular}{|c|c|c|c|}
\hline & & Frequency & Percent \\
\hline \multirow{12}{*}{ 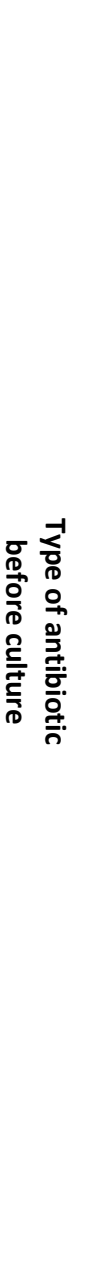 } & No & 19 & 31.7 \\
\hline & Cefepime & 2 & 3.4 \\
\hline & Cefoperazone (Cefobid) & 8 & 13.3 \\
\hline & Cefotaxime (Cefotaxim) & 1 & 1.7 \\
\hline & Ceftriaxone & 2 & 3.3 \\
\hline & Cefotaxime (Claforan) & 19 & 31.6 \\
\hline & ceftazidime(Fortum) & 1 & 1.7 \\
\hline & (Ampicillin, sulbactam) unasyn & 26 & 43.3 \\
\hline & Unasyn and Cefobid & 1 & 1.7 \\
\hline & Vancomycin & 5 & 8.3 \\
\hline & (azithromycin) Zithrodose & 1 & 1.7 \\
\hline & (azithromycin) Zithromax & 1 & 1.7 \\
\hline \multirow{7}{*}{ 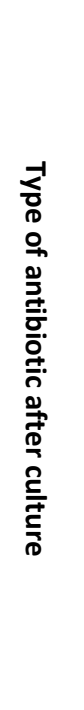 } & No & 57 & 95.0 \\
\hline & Cefepime & 1 & 1.7 \\
\hline & Meropenem (Meronem) & 1 & 1.7 \\
\hline & Meronem & 1 & 1.7 \\
\hline & ceftazidime (Fortum) & 1 & 1.7 \\
\hline & Vancomycin & 1 & 1.7 \\
\hline & Total & 60 & 100.0 \\
\hline
\end{tabular}


Table 3: Fate and remission rate in comparison between infected and non-infected group

\begin{tabular}{|c|c|c|c|c|c|c|c|}
\hline & & & & & & & \\
\hline & & & No & Infected & Toldi & $n$ & $r$ \\
\hline & Activity loading to & Count & 0 & 3 & 3 & & \\
\hline & renal Tallure & $\%$ within GROUP & $0.0 \%$ & $5.0 \%$ & $3.3 \%$ & & \\
\hline & & Count & 0 & 1 & 1 & & \\
\hline & & $\%$ within GROUP & $0.0 \%$ & $1.7 \%$ & $1.1 \%$ & & \\
\hline & & Count & 0 & 1 & 1 & & \\
\hline & Thrombosis & $\%$ within GROUP & $0.0 \%$ & $1.7 \%$ & $1.1 \%$ & & \\
\hline & & Count & 30 & 55 & 85 & & \\
\hline & & $\%$ within GROUP & $100 \%$ & $91.7 \%$ & $94.4 \%$ & & \\
\hline & & Count & 0 & 5 & 5 & & \\
\hline 首 & Unfavorable & $\%$ within GROUP & $0.0 \%$ & $8.3 \%$ & 5.5 & & \\
\hline$\frac{2}{3}$ & & Count & 30 & 55 & 85 & & \\
\hline & Favorable & $\%$ within GROUP & $100 \%$ & 91.7 & 94.5 & & \\
\hline & & Count & 30 & 60 & 90 & & \\
\hline & Total & $\%$ within GROUP & $100 . \%$ & $100.0 \%$ & $100.0 \%$ & & \\
\hline
\end{tabular}

\section{Discussion}

Episodes of nephrotic syndrome are often temporally associated with occurrence of infection. As glucocorticoids and other immuno-suppressive drugs is the mainstay of therapy, infection occurring during therapy is well-known and is partially explainable by the relative immunocompromised state. However, infection often occurs even when the child is not on glucocorticoid therapy [5]. Webb and his colleagues 2016 reported that awareness of common pathogens responsible for infections in children with INS is vital to guide initial antimicrobial therapy [6]. The current study showed that there is high rate of infection among admitted cases with activity $(66.6 \%)$, and we found the high incidence of infection due to admission of cases during summer with high rate of gastroenteritis and inclusion of URTI.

Other studies reported different rates of infection in NS cases; for example [7] Krishnan, 2017 mentioned that a total of 246 children with nephrotic syndrome were enrolled, of whom 46 children developed 48 (19.6\%) episodes of infections. Ajayan 2013 [8] mentioned that
Major infections were defined as; those that are scattered, hit deep organs, need hospitalization or potentially fatal. The incidence of these infections was $36.6 \%$.

According to Soares, 2014 [9] there was a significant decrease in the incidence and transmission of infection nowadays in Brazil because of the wider vaccination administration to children. This may explain less incidence of this infection as a cause of NS; While Alwadhi 2004 [5] reported that of the 76 activities, 8 were excluded from analysis. Of the remaining 68 nephrotic attacks in 60 patients, there was clue of infection in 57 attacks $(83.8 \%)$.

In our study; The most common type of infection was gastroenteritis (GE) (25\%) of infected cases and 2nd most common Then Urinary tract infection (UTI) $(21.7 \%)$, While Pneumonia represented (15\%) of infection. According to Uwaezuoke, 2015 [4] and Krishnan 2017 [7] Pneumonia $(41.7 \%)$ was the most common infection, urinary tract infection (25\%), septicemia $(16.7 \%)$, followed by spontaneous bacterial peritonitis (8.3\%), Ajayan P.2013 [8] mentioned that within the major infections, peritonitis 
plus pneumonia were for $72.9 \%$, while urinary tract infections plus cellulitis were $16.2 \%$.

In a U.S. retrospective study focusing on 1ry peritonitis in INS, S. pneumoniae was the most common organism isolated, about for $38 \%$ of patients, with Gram-negative bacteria only $3 \%$ of patient. And $27 \%$ of patients were with negative culture results. Another article studying peritonitis in childhood NS found that S. pneumoniae was the most common pathogen identified $(50 \%)$, but that Escherichia coli accounted for $25 \%$ of cases, and $16 \%$ of cases were culture-negative [6]. Our study also showed that there was no significant pathogen in $(61.66 \%)$, the is E.coli $(25 \%)$, followed by Shigella $(6.66 \%)$, pneumococci $(3.33 \%)$, klebsiella (1.66\%) and proteus $(1.66 \%)$. Of all infected cases, matching our results Alwadhi, 2004 [5] mentioned that of [13] UTI, [11] had Gram negative organisms, namely Escherichia-coli and Klebsiella. Enterococcs was isolated in the other two. Urinary tract infection was asymptomatic in $(77 \%)$ of cases.

Three out of nine cases of peritonitis showed Gram negative bacilli on staining of peritoneal fluid examination. However, no organism was isolated from any ascetic fluid samples. Our study showed that there was high statistical significant difference as regarding CRP results between infected and non-infected group before and after treatment. And show that $(60 \%)$ of CRP results in infected group are negative that explain culture results that no pathogen in $(66.6 \%)$ be viral infection; Therefore, sCRP may serve as a reliable marker of inflammation in this setting according to Wasilewska, 2007 [10]. Increases in CRP values are nonspecific and should not be interpreted without a complete clinical evaluation. Conventional assays for CRP are not sensitive enough and, therefore, not reliable in healthy subjects Conventional CRP assays are indicated for use for evaluation of infection, the children with

relapse of nephrotic syndrome showed a significant increase in CRP level. In our study, 36/60 (60\%) oF infected cases had CRP negative and $40 \%$ were CRP positive. As regarding TLC $26.7 \%$ were neutrophilic and $21.7 \%$ show Lymphocytosis.

As regarding culture results $37 / 60(61.6 \%)$ of cases show no growth; so $(60 \%)$ of patients had viral infection. According to Uwaezuoke, 2015 [4] It is found that at least half of activities were triggered by a viral upper respiratory tract infection which may be associated to non-specific host response to infection.

In our study that there was statistical significant affection of hemoglobin and serum creatinine in infected than non-infected group. Also, there is no significant difference between both groups regarding steroid response whether infected or not. Also, in our study No statistical significant differences as regarding type of steroid used to induce remission, According to Larkins, 2016 [11] the majority of children who relapse continue to respond to steroids throughout their subsequent course, and the longterm prognosis, including maintenance of normal kidney function, is good, While, Gulati, 2011 [12] mentioned that daily doses of steroid, during inter-current infections, significantly decrease activity frequencies and number of children with frequently relapsing NS.

The decline in activity times in the study was mainly because of decreasing number of infection-triggered relapses. $70 \%$ of activities in the intervention patients and $74 \%$ in the controls were preceded by infections, mainly of the upper respiratory tract. The mechanism by which infections induce activities of NS is vague but might be due to the up-regulation of $\mathrm{T}$ cells plus cytokine induced rise in proteinurea. But Jamin, 2015 [13] mentioned that the clinical course of INS is largely dependent upon the response to steroids.

Relapses are frequent after withdrawal of steroid treatment, with up to $60 \%$ of patients being steroiddependent. In our study, all cases receiving cyclosporine presented with active disease. While not all cases receiving azathioprine presented with active disease that means that cyclosporine precipitate infection.

According to Soares, 2014 [9] immunosuppressant in children with chronic infections may lead to the deterioration of the infection and of the general clinical state. So; most regimes of management of children with NS suggest screening of infections, because these may need specific treatment. According to [4] Uwaezuoke, 2015.

The new findings however do not preclude the requirement for the use of chemotherapy drugs like steroids or cycholsporins as these drugs also alter podocyte function as well as structure. So , the KDIGO recommendation of prescribing daily steroids in activities of upper respiratory infections still a golden intervention targeting reduction the risk of activity.

In our study the most frequently used antibiotic before culture is Ampicillin, sulbactam followed by cefotaxime and after culture Cefepime, Meropenem, ceftazidime and Vancomycin are the most. There is high statistical significant difference between both groups regarding antiviral response between infected and non-infected group. There was $(21.7 \%)$ of infected cases received antiviral treatment, According to Yildirim 2015 [14], it appears that penicillin, ampicillin, or cefuroxime should be adequate to treat children hospitalized with pneumonia due to pneumococcal isolates .Oral therapy with amoxicillin, amoxicillin-clavulanate, or cefuroxime also should be effective for 1st outpatient management or when ending therapy once the child has resolution of signs and symptoms following parenteral treatment.

While, Hogan, 2015 [15] stated that Antiviral treatment leads to a complete remission of the NS, with no requirement for steroid. In our study, there is high significant difference between both groups regarding plasma transfusion between infected and non-infected group and no statistical significant difference regarding albumin infusion this comes into agreement with Alwadhi, 2004 [5] who reported that Children with infection had significantly lower serum albumin compared to noninfected children. In our study there is $(91.7 \%)$ of infected group had fair out come and (8.3\%) of cases had 
unfavorable outcome as following: (5\%) of cases show Activity leading to renal failure, $(1.7 \%)$ of cases died from pneumonia and (1.7\%) of cases show thrombosis. Krishnan, 2017 [7] mentioned that there was no mortality in his study.

While Uwaezuoke, 2015 [4] reported that only $20 \%$ respond to the medication with a more risk of developing end-stage renal failure. Lin JN, 2016 [16] reported that the long-term prognosis of NS is mostly good; but, its characteristics and related treatment can greatly affect childhood development and quality of life in those patients. Resistant cases in some patients may deteriorate up to endstage renal disease.

Larkins, 2016 [11] Reported that without therapy, NS in children comes with high risk of mortality, especially of bacterial infection. In the past before the use of steroid and antibiotics, $40 \%$ of patients were lost, with $50 \%$ of these losses were due to infection.

\section{Conclusion}

We concluded that $66.6 \%$ of admitted children with NS with relapse were due to infection, the most common type of infection is gastroenteritis, followed by Urinary tract infection, upper respiratory tract infection and Chest infection. We concluded also that Viral infection represents high rate of infected cases depending on clinical and laboratory data and the most common type of bacteria after culture is E.coli, Shigella, pneumococci, klebsiella and proteus.

\section{References}

1. Jamin A, Dehoux L, Dossier C, Fila M, Heming N, Monteiro R, and Deschênes G. Toll-like receptor 3 expression and function in childhood idiopathic nephrotic syndrome, Clin Exp Immunol.. 2015, 182(3): 332-345

2. Rheault MN, Zhang L, Selewski DT, Kallash M, Tran CL, Seamon K, Katsoufis C, Ashoor I, Hernandez J, Supe-Markovina K, D'Alessandri-Silva C, DeJesusGonzalez N, Vasylyeva TL, Formeck C, Woll C, Gbadegesin R, Geier P, Devarajan P,Carpenter SL, Kerlin
BA, Smoyer WE; Midwest Pediatric Nephrology Consortium. AKI in Children Hospitalized with Nephrotic Syndrome. Clin J Am Soc Nephrol. 2015, 10(12):2110-8. 3. Kidney International Supplements. Steroid-sensitive nephrotic syndrome in children.

Kidney Int Suppl. 2011, 2(2): 163-171.

4. Uwaezuoke $\mathrm{S}$ N: Steroid-sensitive nephrotic syndrome in children: triggers of relapse and evolving hypotheses on pathogenesis, Ital J Pediatr. 2015, 41: 19.

5. Alwadhi RK, Mathew JL, Rath B. Clinical profile of children with nephrotic syndrome not on glucorticoid therapy, but presenting with infection.

J Paediatr Child Health. 2004, 40 (1-2):28-32.

6. Webb NJ, Frew E, Brettell EA, Milford DV, Bockenhauer D, Saleem MA, Christian M, Hall AS, Koziell A, Maxwell H, Hegde1 S, Finlay ER, Gilbert RD, Booth J, Jones C, McKeever K, Cook W, Ives NJ. (Short course daily prednisolone therapy during an upper respiratory tract infection in children with relapsing steroid-sensitive nephrotic syndrome).

Trials;2014, 15:147.

7. Krishnan C, Rajesh T. V, Shashidhara H. J, Jayakrishnan M. P, Geeta M. G. Major infections in children with nephrotic syndrome International Journal of Contemporary Pediatrics 2017, (2):346-35.

8. Ajayan P, Krishnamurthy S, Biswal N, Mandal J. Clinical spectrum and predictive risk factors of major infections in hospitalizedchildren with nephrotic syndrome. Indian Pediatr. 2013, 50 (8):779-81.

9. Yildirim I, Shea KM, and Pelton S. Pneumococcal Disease in the Era of Pneumococcal Conjugate Vaccine Infect Dis Clin North Am.2015, 29(4): 679-697

10. Hogan J, Fila M, Baudouin V, Peuchmaur M, and Deschênes G, Niel O. Cytomegalovirus infection can mimic genetic nephrotic syndrome: a case report. 2015, 22; 16(1):156

11. Lin JN, Lin CL, Yang CH, Lin MC, Lai CH, Lin $\mathrm{HH}$, Kao CH. Risk of Nephrotic Syndrome following Enteroviral Infection in Children: A Nationwide Retrospective Cohort Study. PLoS One; 2016, 11(8).

Larkins N, Kim S, Craig J, Hodson E. (Steroid-sensitive nephrotic syndrome: an evidence-based update of immunosuppressive treatment in children). Arch Dis Child. 2015, 101(4):404-8.

\section{Declarations}

Ethics approval and consent to participate

This study protocol and the consents were approved and deemed sufficient by "The Postgraduate Clinical Research and Ethical Committee of Pediatric Department, Faculty of Medicine, Zagazig University.” And informed written consent was obtained in every case from their legal guardians.

\section{Funding}

The authors declare that they didn't receive any financial support from agencies or others.

\section{Conflict of interest}

No

\section{Acknowledgements}

We would like to thank all patients and their family members for their valuable contributions to the study. 